\section{SINGLE-CENTER RETROSPECTIVE COHORT OF ALL PATIENTS SUSPECTED TO HAVE IMMUNOTHERAPY- MEDIATED DIARRHEA AND COLITIS}

Vanya Aggarwal*, Ella LePage, Andrew Faucheux, Hiral Patel, Gregory Russell, Eric Olson, Jared Rejeski, Thomas Lycan. Wake Forest School of Medicine, Winston-Salem, NC, United States

Background Immune-mediated diarrhea or colitis (IDC) is a potentially serious adverse event which can occur in up to $10 \%$ of patients receiving an immune checkpoint inhibitor (ICIs), but not all episodes of diarrhea among these patients are immune-mediated. ${ }^{1}$ There is a paucity of research regarding the diagnosis and management of this common symptom among patients receiving an ICI.

Methods We collected retrospective clinical data for all patients who received at least one dose of an ICI for any cancer diagnosis $(n=2,120)$ and subsequently underwent a diagnostic workup for acute diarrhea with stool testing for either C. difficile or a gastrointestinal pathogen panel $(n=223)$ at any point between $1 / 1 / 13$ to $3 / 17 / 21$. We compared patients who had IDC "ruled out" to those who had confirmed IDC using Fisher's exact test for categorical variables and either independent samples t-test or Wilcoxon two-sample tests for interval variables. The Kaplan-Meier method was used to estimate progression-free and overall survival time. A two-sided alpha of 0.05 was utilized in determining which relationships might be significant.

Results Thirty-seven percent had ICIs deferred upon symptom onset, and $28 \%$ received systemic steroids. Patients receiving an ICI who developed diarrhea were $2.14 \mathrm{x}$ more likely to have a different etiology for their symptoms $(n=152,68 \%)$ than IDC. Patients who had IDC ruled out were more likely to be female $(47 \%, \mathrm{p} 0.029)$ and have at least one comorbidity $(93 \%, \mathrm{p}$ 0.028). Patients with confirmed IDC were more likely to have peptic ulcer disease $(4 \%, \mathrm{p} 0.031)$, to have received ipilimumab $(24 \%, \mathrm{p}<0.0001)$ or $>1$ ICI concurrently $23 \%, \mathrm{p}<0.001)$, and to have a shorter time since last dose of immunotherapy to onset of symptoms $(12$ vs. 26 days, $p$ $<0.0001)$. There were no differences in age, race, ethnicity, prior cancer therapies, types of other comorbidities, symptoms, presence of other adverse events, number of ICI cycles prior to symptom onset, or performance status. Progression-free survival was longer among patients with confirmed IDC ( $p$ 0.003 ). Overall survival was longer among patients with confirmed IDC (p 0.021) (figure 1).

Conclusions Diarrhea is often due to another etiology besides IDC, especially among patients who have onset of symptoms over 2 weeks after receiving an ICI other than ipilimumab. If ipilimumab or two ICIs are used concurrently, it is warranted to have increased suspicion for IDC especially with rapid progression of symptoms. This dataset provides additional evidence that confirmed IDC may be associated with prolonged progression-free and overall survival.

\section{REFERENCE}

1. Wang $Y$, Zhou $S$, Yang $F$, et al. Treatment-related adverse events of PD-1 and PD-L1 inhibitors in clinical trials: a systematic review and meta-analysis. JAMA Oncol 2019;5(7):1008-1019. doi:10.1001/jamaoncol.2019.0393

Ethics Approval The study was approved by Wake Forest Baptist Health Ethics Board, approval number \#IRB00044126.

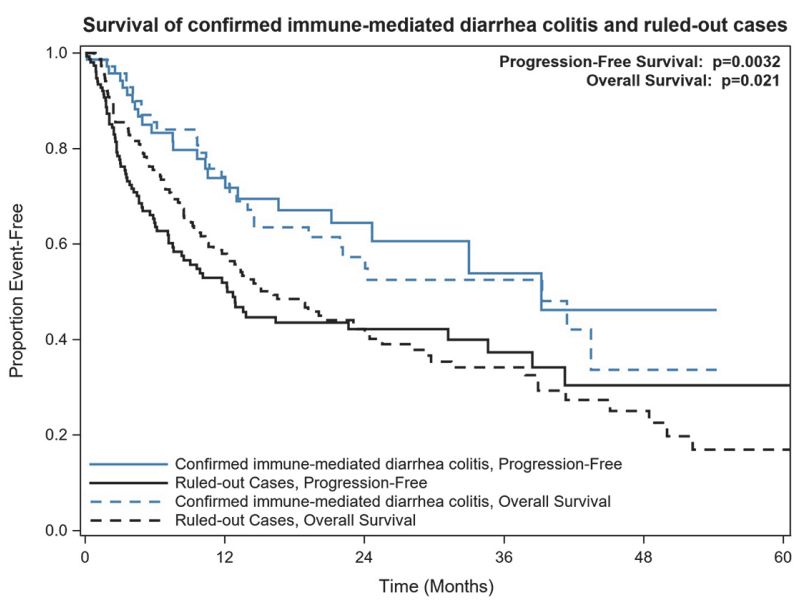

Abstract 815 Figure 1 Survival of confirmed IDC and ruled-out cases

http://dx.doi.org/10.1136/jitc-2021-SITC2021.815 